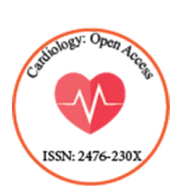

ISSN: 2476-230X

\title{
Role of Natriuretic Terminal Pro B-type Natriuretic Peptide (NT-Pro-BNP) in Diagnosis of Heart Failure in Acute Exacerbation of Chronic Obstructive Pulmonary Disease (COPD), Retrospective Cohort Study Conducted in Karachi, Pakistan
}

\author{
Khalida Soomro ${ }^{1 *}$, Muhammad Ali² \\ ${ }^{1}$ Consultant Cardiologist $7^{\text {th }}$ Day Karachi Adventist \\ Hospital, Karachi, Pakistan
}

${ }^{2} \mathrm{MBBS}$, Demonstrator of Pathology, Bilawal Medical College, LUMHS Jamshoro

\author{
"Corresponding author \\ Khalida Soomro, Consultant Cardiologist $7^{\text {th }}$ day Karachi Adventist Hospital,
} Pakistan.

Submitted: 18 Dec 2021; Accepted: 30 Dec 2021; Published: 19 Jan 2022

Citation: Khalida Soomro, Muhammad Ali (2022) Role of Nt-Pro-Bnp in Diagnosis of Heart Failure in Acute Exacerbation of Copd, Retrospective Cohort Study Conducted in Karachi, Pakistan. Cardio Open, 7(1): 174-179.

\begin{abstract}
Introduction: COPD and heart failure effecting millions of people in the world where as combinedly becomes deadlier and affecting the major population of different regions of world, also increasing the rate of morbidity and mortality. Diagnosis of heart failure in COPD patients or COPD in heart failure patient is very difficult for cardiologist and pulmonologist.

Objective: Acute exacerbation of COPD concomitant with Heart failure is among the diseases responsible for increasing the Mortality, morbidity, economic burden on patients, health care system and to see the impact on gender-based incidence Early use of NT-Pro-BNP is evaluated in COPD patients for diagnosis of heart failure in Pakistan due to socio-cultural and economical differences this study was conducted.
\end{abstract}

\begin{abstract}
Methods: The study was conducted in 7th Day Karachi Adventist hospital from Nov 2020 to Nov 2021, retrospective cohort study in admitted patients with acute exacerbation of COPD. Qualitative variables including gender, age groups, smoking status, symptoms and clinical finding were recorded. Data was entered and analyzed in statistical package for social sciences (SPSS) version 22. Quantitative variables including age in years, duration of COPD, NT-Pro-BNP level and ejection fraction was presented in mean and standard deviation. For the determination of association between qualitative variables chi-square test was used.
\end{abstract}

Results: In our study heart failure was reported in 89 (85.5\%) patients of AECOPD on the basis of Pro -BNP level with Heart Failure \& COPD without Heart Failure in 15 (14.4\%) patients by using NT-Pro-BNP as a marker of heart Failure. independent sample was used for calculating mean difference (8042.2),95\%CI (1060.7-23.0) and P-value 0.02 *between patients of COPD with heart failure and without HF P-value shows significant difference between Pro BNP level of COPD with heart failure and without Heart failure. Gender of Patients with COPD was male in 61 (58.6\%) patients and female in 43 (41.3\%). Mean age of the patient with acute exacerbation of COPD was 70.0 11.2 (40-98) years. Age distribution of patients with acute exacerbation of COPD in male vs female was in age group <50 years was 4 (3.8\%) vs 1 (0.9\%), in age group 50-75 years was 50 (48\%) vs 22 (21.1\%), and in age group <75 years was $20(19.2 \%)$ vs 7 (6.7\%) shows male patients more suffering than women in all age groups. On history and clinical examination Symptoms of shortness of breathing and Coughing were more in male then female. Mean ejection fraction of patients with acute exacerbation of COPD without HF minimum E.F was $40 \%$ and maximum was $70 \%$ with mean $26 \%$. COPD with HF minimum E.F was $20 \%$ and maximum was $70 \%$ with mean $52 \% \pm 16.6$ (20-70\%). Mean Pro-BNP level of patients with acute exacerbation of COPD was $7498 \pm 12221$ (17-36000) $\mathrm{pg} / \mathrm{ml}$. Chi-square test value was 0.122 and P-value was 0.7.

Conclusion: It was concluded from this study that the level of NT-Pro-BNP in patients with acute Exacerbation of COPD. diagnosed with Heart Failure was significantly high as compared to patient of acute exacerbation without Heart Failure especially high in males than females. Early diagnosis of heart failure in COPD patients will be helpful in early initiation of heart failure, medication, decreasing the hospital admissions, hospital stay and cost burden on patients and health care system of low socio-economical countries.

Keywords: Chronic Obstructive Pulmonary Disease (COPD), Acute Exacerbation of Chronic Obstructive Pulmonary Disease (AECOPD), Natriuretic Terminal Pro B-type Natriuretic Peptide (NT-Pro-BNP), Heart Failure (HF), Ejection Fraction (EF), Cardiovascular Diseases (CVD) 


\section{Introduction}

COPD and heart failure, both diseases alone are affecting millions of people in the world where as combinedly becomes deadlier and affecting the major population of different regions of world, also increasing the rate of morbidity and mortality. COPD is playing an important role in increasing the risk and progression of cardiovascular diseases [1]. Left heart failure is more common in COPD patients and diagnosed in every fifth patient of the COPD. Similarly, COPD is more diagnosed in one out of three patients with Heart Failure. Several research studies are conducted on association of cardiovascular and COPD risk factors, they reported the following common risk factors; Increasing age, Male gender, Family history of coronary artery disease (CAD), Diabetes mellitus, Dyslipidemia, Cigarette Smoking.

Diagnosis of heart failure in COPD patients or COPD in heart failure patient is very difficult for cardiologist and pulmonologist. As early diagnosis of COPD in heart failure patient can be helpful in reversing concomitant obstructive ventilation disorders hence have impact on the prognosis of the patient. Different diagnostic techniques at different times are used in COPD patients such as X-ray chest, transthoracic echocardiography, cardiac magnetic resonance imaging, as cardiopulmonary exercise test or elevation of CRP, troponin or NT-Pro-BNP are used for diagnosis of CVD. NT-Pro-BNP is nowadays use for COPD patients for early prediction of heart failure [2-4].

B-type natriuretic peptide (BNP) and N-terminal pro b-type natriuretic peptide (NT-pro-BNP) are peptide (small proteins) that are either hormones or part of the peptide that contained the hormone at one time. They are continually produced in small quantities in the heart and released in larger quantities when the heart senses that it needs to work harder. Subsequently, the heart muscle is stretched and works hard to pump blood under normal resting condition [5-11]. When the left ventricle of the heart is having difficulty pumping sufficient amounts of blood to the body, the concentrations of BNP and NT-pro-BNP produced can increase markedly and increase in circulating BNP or NT-proBNP will reflect this diminished capacity to deliver oxygenated blood to the body. NT-Pro-BNP $>500 \mathrm{pg} / \mathrm{mL}$ are clear indicator of heart failure, whereas $<100 \mathrm{pg} / \mathrm{mL}$ indicates the differential diagnoses of pulmonary disorders. Between these values, diagnostic accuracy of NT-Pro-BNP is limited in detection of biventricular and right heart failure [12-15]. NT-Pro-BNP level is more accurate in predicting systolic left ventricular dysfunction as compared diastolic left ventricular dysfunction $[16,17]$.

\section{Aims and Objective}

NT-Pro-BNP level as a bio marker is used in differential diagnosis of dyspnea in patients of COPD which compares the level of NT-Pro-BNP in patients presented with AECOPD patients and used for diagnosis or exclusion of heart failure. CVD is responsible for increasing the hospital admission stay in hospital and enforcing economic burden of patients on health care system. NT-Pro-BNP is useful in early diagnosis of heart failure and initiation of heart failure medications, decreasing the hospital admission, decreasing the hospital stay, decreasing the cost burden on patients and health care system. [18-20] Especially due to non-availability and limited data internationally and in Pakistan regarding the gender-based comparison of NT-Pro-BNP in patients in AECOPD with Heart Failure enforces the designing of current study.

\section{Method}

The study was conducted in 7th Day Karachi Adventist hospital from Nov 2020 to Nov 2021. Admitted patients were enrolled in this single-center, retrospective cohort study. Qualitative variables including gender, age groups, smoking status, shortness of breath, persistent coughing or wheezing, increase sputum production, ankle swelling, fatigue, and outcome were recorded with the help of Questionnaire and Clinical examination. Oral informed consent was approved by the ethics commission of the hospital for these patients. (COPD with \& without HF) were presented in frequency and percentages. Data was entered and analyzed in statistical package for social sciences (SPSS) version 22. Quantitative variables including age in years, duration of COPD, NT-Pro-BNP level and ejection fraction was presented in mean and standard deviation. For the determination of association between qualitative variables chi-square test was used and the level of significant was $95 \%$ taking $\mathrm{p}$-value $<0.05$ as significant. Mean difference and $95 \%$ confidence interval (CI) was calculated for association between NT-Pro-BNP and heart failure.

\section{Result}

A total of 104 patients having acute exacerbation of COPD and admitted in 7th Day Karachi Adventist Hospital were evaluated for the risk of Heart Failure by using NT-Pro-BNP as a marker of heart Failure. Gender of Patients with COPD was male in $61(58.6 \%)$ patients and female in $43(41.3 \%)$. Mean age of the patient with acute exacerbation of COPD was 70.0 \pm 11.2 (4098) years. Age distribution of patients with acute exacerbation of COPD in male vs female was in age group $<50$ years was 4 $(3.8 \%)$ vs $1(0.9 \%)$, in age group 50-75 years was $50(48 \%)$ vs $22(21.1 \%)$, and in age group $<75$ years was $20(19.2 \%)$ vs 7 $(6.7 \%)$ shows male patients more suffering than women in all age groups.

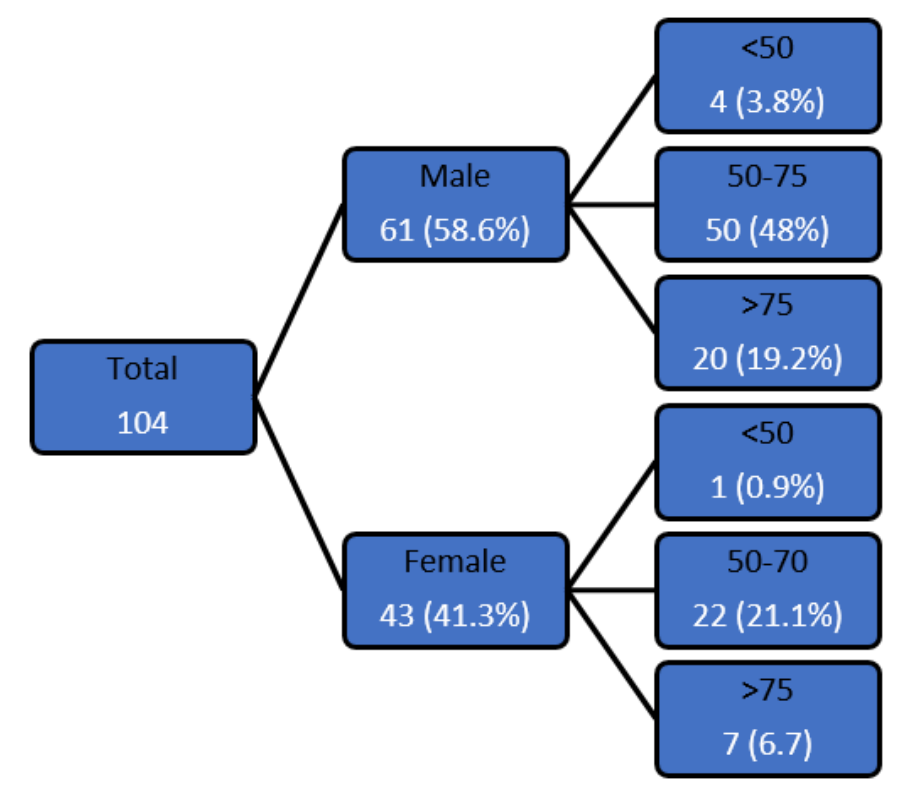

Figure 1: Age Group of Patients

Patient with COPD and Heart Failure out of 104 patients were $89(85.5 \%)$ and Heart Failure was absent in COPD in $15(14.4 \%)$ patients. Gender of Patients with COPD and Heart Failure was male in $52(50 \%)$ vs female in $37(35.5 \%)$ and COPD without Heart Failure was male in $9(8.6 \%)$ vs female in $6(5.7 \%)$. 


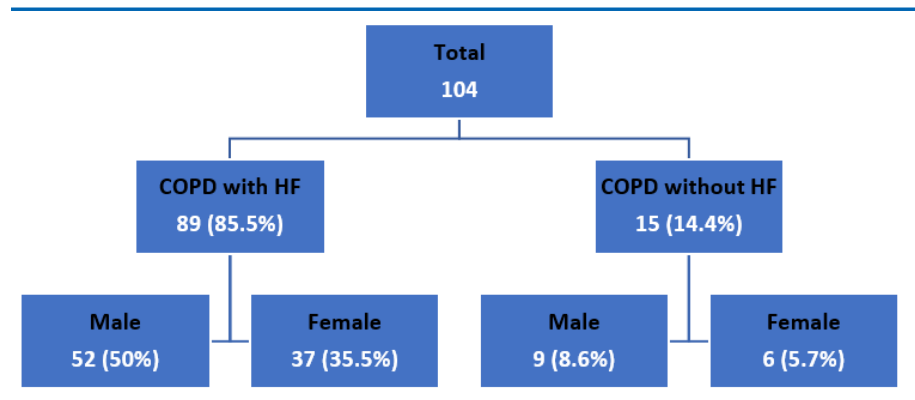

Age Distribution of patient with COPD and Heart Failure in male's vs females in age group $<50$ years was $6(5.8 \%)$ vs 6 $(5.8 \%)$, in age group 50-70 years was $28(26.9 \%)$ vs $20(19.5 \%)$ and in age group $>70$ years was $18(17.3 \%)$ vs $11(10.5 \%)$ and COPD without Heart Failure in male's vs females in age group $<50$ years was $1(0.9 \%)$ vs $0(0 \%)$, in age group $50-70$ years was $3(2.8 \%)$ vs $2(1.9 \%)$ and in age group $>70$ years was $6(5.8 \%)$ vs $4(3.8 \%)$. Chi-square test value was 0.122 and $\mathrm{P}$-value was 0.7 .

Figure 2: Stratification of Outcomes with Gender of Patients

Table 1: Stratification of Outcomes with Gender of Patients

\begin{tabular}{|c|c|c|c|c|c|c|c|}
\hline \multirow{3}{*}{$\begin{array}{l}\text { Gender } \\
\text { Age }\end{array}$} & \multicolumn{7}{|c|}{ Outcomes } \\
\hline & \multicolumn{3}{|l|}{ Male } & \multicolumn{3}{|l|}{ Female } & \multirow[t]{2}{*}{ Total } \\
\hline & $<50$ & $50-70$ & $>70$ & $<50$ & $50-70$ & $>70$ & \\
\hline COPD with HF & $6(5.8 \%)$ & $28(26.9 \%)$ & $18(17.3 \%)$ & $6(5.8 \%)$ & $20(19.2 \%)$ & $11(10.5 \%)$ & $89(85.5 \%)$ \\
\hline COPD without HF & $1(0.9 \%)$ & $3(2.8 \%)$ & $6(5.8 \%)$ & $0(0 \%)$ & $2(1.9 \%)$ & $4(3.8 \%)$ & $15(14.4 \%)$ \\
\hline Total & \multicolumn{3}{|c|}{$61(58.6 \%)$} & \multicolumn{3}{|c|}{$43(41.3 \%)$} & 104 \\
\hline \multicolumn{8}{|l|}{ Chi-square Test } \\
\hline Chi-square valve & \multicolumn{7}{|l|}{0.122} \\
\hline P-Value & \multicolumn{7}{|l|}{0.7} \\
\hline
\end{tabular}

On history and clinical examination shortness of breathing with acute exacerbation of COPD was present in 69 patients, out of them 58 were male's vs 41 were females. Frequency of Ankle Swelling with acute exacerbation of COPD was present in 15 patients out of them 9 were male's vs 6 were females. Fatigue with acute exacerbation of COPD was present in 98 patients out of them 56 were male's vs 42 were females and absent in 6 patients. Persistent Coughing and wheezing in patients with acute exacerbation of COPD was present in 88 patients out of them 48 were male's vs 40 were females and absent in 16 patients.

Table 2: Symptoms of the Heart Failure and COPD

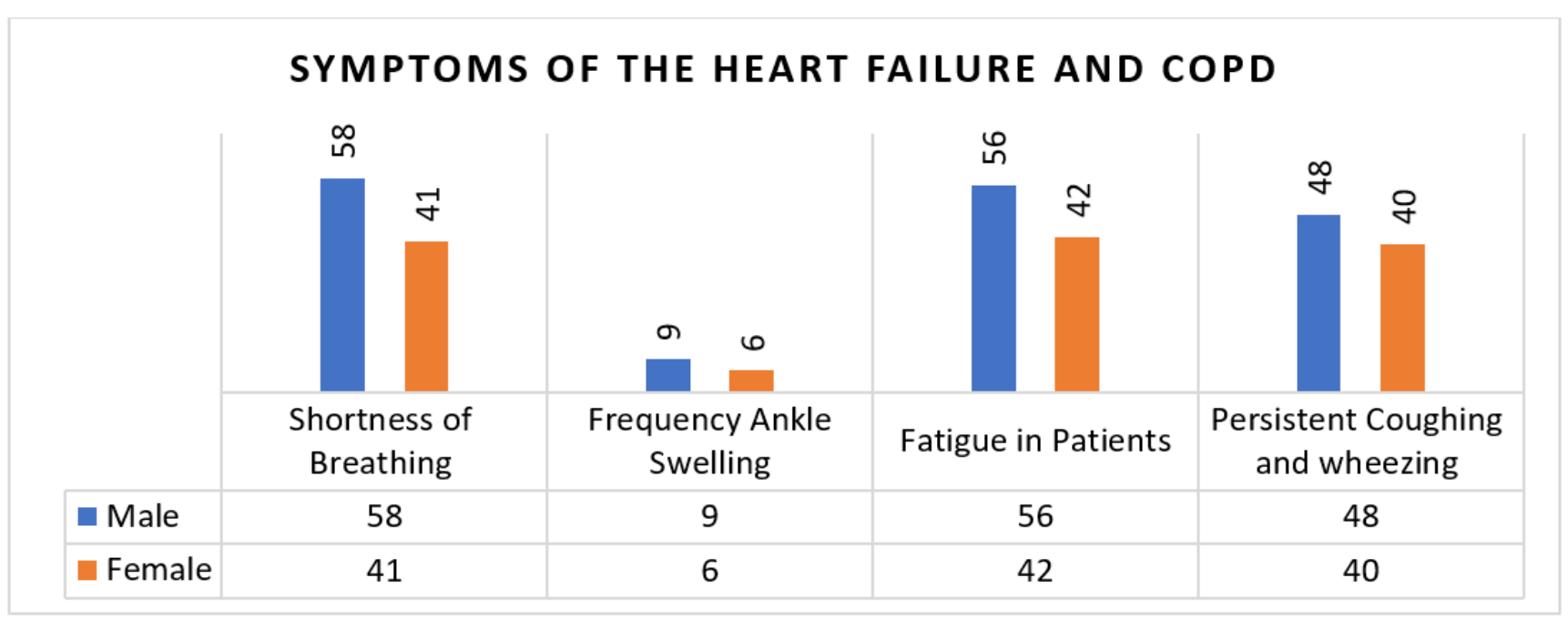


Table 3: Association Between Heart Failure and NT-Pro-BNP

\begin{tabular}{|l|l|l|}
\hline NT-Pro-BNP & CODP with HF & CODP without HF \\
\hline N & 89 & 15 \\
\hline Minimum & 138.5 & 17.2 \\
\hline Maximum & 36000 & 1200 \\
\hline Mean & 8010.6 & 165.4 \\
\hline Standard Deviation & 11000.9 & 354 \\
\hline Independent Sample T Test & & \\
\hline T-test value & 2.180 & \\
\hline Mean Difference & 7840.2 & \\
\hline 95\% CI & $1010-14-24$ & \\
\hline P-Value & $0.04 *$ & \\
\hline
\end{tabular}

Mean Pro-BNP level of patients with acute exacerbation of COPD was $7498 \pm 12221$ (17-36000) pg/ml.

Table 4: Descriptive Statistics of NT-Pro-BNP level

\begin{tabular}{|l|l|}
\hline NT-Pro-BNP Level $(\mathbf{p g} / \mathbf{m l})$ \\
\hline Total Number & 104 \\
\hline Minimum & 17 \\
\hline Maximum & 36000 \\
\hline Mean & 7498 \\
\hline Standard Deviation & 12221.8 \\
\hline
\end{tabular}

Among the 104 patients included in our study, a significantly higher incidence of left ventricular dysfunction was observed in COPD with Heart Failure Patients. Mean ejection fraction of patients with acute exacerbation of COPD without HF E.F was

Table 5: Descriptive Statistics of Ejection Fraction

\begin{tabular}{|l|l|l|}
\hline \multirow{2}{*}{ Total Number } & \multicolumn{2}{|l|}{ Ejection Fraction (\%) } \\
\cline { 2 - 3 } & 104 & COPD without HF $14.4 \%$ \\
\cline { 2 - 3 } & COPD with HF 85.5\% & $40 \%$ \\
\hline Minimum & $20 \%$ & $70 \%$ \\
\hline Maximum & $70 \%$ & $26 \%$ \\
\hline Mean & $52 \%$ & \multicolumn{2}{|l}{} \\
\hline Standard Deviation & $16.6 \%$ & \\
\hline
\end{tabular}

\section{Discussion}

Both organ system lungs and heart should be considered at same time for clinical and diagnostic evaluation for early diagnosis and appropriate management of heart failure in patient with COPD. the past history can be helpful in detection of heart failure [18, 19]. On clinical examination such as apical and ventral wheezing seen in COPD only and alveolar edema developed because of decompensation of left heart can results in fine crackles and wheezing in lungs lower parts. Conventional X-ray is not much helpful due to chronic structural changes. Tests for BNP and NTpro-BNP measure their levels in the blood in order to detect and evaluate heart failure. The two tests are not interchangeable and should not be used together.

In our study, we found that the incidence of COPD with Heart Failure was higher than absent heart failure in patients. these Cardio Open, 2022
$40 \%$ minimum and $70 \%$ maximum with mean $26 \%$. In COPD with HF minimum E.F was $20 \%$ and maximum was $70 \%$ with mean $52 \% \pm 16.6(20-70 \%)$. findings corelates with another study which used the NT-ProBNP level in diagnosis of heart failure in patients presented with exacerbation COPD. Sensitivity of NT-Pro-BNP was $94.4 \%$, specificity $68.2 \%$ with diagnostic accuracy of $74.3 \%$.

Shaikh k and Ahmed M, study on significance of NT-Pro -BNP in patients of heart failure with acute dyspnea. our study reports 104 COPD presented with dyspnea where as they selected $100 \%$ patients of Dyspnea, they reported the $14.4 \%$ patients with heart failure and our study reported $85.5 \%$ patients with heart failure. similar to our study mean Pro BNP level in patients of COPD with heart failure was high $10918 \pm 1228 \mathrm{pg} / \mathrm{ml}$ and low in patients of COPD without Heart failure $451 \pm 100 \mathrm{pg} / \mathrm{ml}$ with significant p-value 0.001 [21].

Adrish M, et al study on significance of NT-Pro-BNP in detec- 
tion of left ventricular dysfunction in patients of COPD. they reported the $62.3 \%$ COPD patients with high NT-PRO-BNP $88.3 \pm 53,3 \mathrm{pg} / \mathrm{ml}$ in COPD patients without heart failure problem and Higher NT-Pro -BNP 1.077.2 $\pm 1.7078 .1 \mathrm{pg} / \mathrm{ml}$ in COPD patients with HF with significant p-Value $<0.0002$ they haven't the gender differences [22].

In one study the overall lifetime risk of HF was fairly comparable between the sexes; estimated at $21 \%$ for men and $20 \%$ for women at age 40 years in the Framingham Heart Study (FHS), and $33 \%$ for men and $29 \%$ for women at age 55 years in the Rotterdam Study which is different from our study.

Sex differences in the epidemiology of HF become apparent when the type of HF is considered. A study by Sidney 5, et al. describes the relation of young age and female gender in COPD patients having significant cardiovascular morbidity [20]. Significant association of smoking is also reported with COPD and cardiovascular problems in their study, this paradox is maybe due to our socio-cultural differences e.g., Smoking is not common in Pakistan in Women. Sex impacts on almost every facet of heart failure, from risk factors to pathophysiology, phenotypes and outcomes including quality of life. The higher risk of heart failure with reduced ejection fraction in men compared to women has been attributed to women predisposition to macrovascular coronary artery disease and myocardial infarction a well-known antecedent to heart failure with reduced ejection fraction. As well as women have a higher level of estrogen, which enhances the level of ACE2 activity and expression in a concentration-dependent manner, upregulates the expression of angiotensin and prompts vasodilation, NO release and reduced smooth muscle cell proliferation inducing a lower rate of myocardial injury in women. Here in Pakistan Sociocultural factors (low awareness, cultural norms, religious beliefs, and societal position) also deter women from physical activities, fueling this phenotype further [23]. Our COPD with HF was comparatively low than in one study conducted in Europe which short, cigarette smoking was independently associated with an $88 \%$ higher risk of HF in women, compared to a $45 \%$ higher risk in men; the higher relative risk in women was somewhat offset by the lower prevalence of smoking in women compared to men as in our country $[20,24]$.

A study by Sussia $\mathrm{S}$, et al. describes the relation of mortality with increasing age and in male gender in COPD patients. In our study age Distribution of patient in COPD with Heart Failure in male's vs females in age group $<50$ years was $6(5.8 \%)$ vs 6 $(5.8 \%)$, in age group 50-70 years was $28(26.9 \%)$ vs $20(19.5 \%)$ and in age group $>70$ years was $18(17.3 \%)$ vs $11(10.5 \%)$ which was age differences in males, gender association is comparable with a study conducted by Ford ES, et al. describes the relation of increasing age, COPD and cardiovascular risk assessment. Which revealed diagnosis of COPD is significantly correlated with 10-year cardiovascular risk assessment having age 55.74 years. So, COPD with Heart Failure is more common in patient with AECOPD then without Heart Failure and incidence was higher in men than women in all age group [25].

\section{Limitations}

Our Study have some limitations due to widespread application of echocardiography with different operators. Next, this study had a single-center design; our findings require validation in further rigorous prospective studies. Thirdly, our study was retrospective in nature and could only speculate the biological relationship between sex differences and Heart Failure with myocardial injury on the basis of our evidence and that of previous studies.

\section{Conclusion}

Our results suggest that the level of NT-Pro-BNP in patients with acute Exacerbation of COPD diagnosed with Heart Failure was significantly high as compared to patient of acute exacerbation of COPD especially in male's vs females, the incidence of Heart Failure in AECOPD is high in patients and gender dependent. Acute Exacerbation COPD concomitant with HF is among the diseases responsible for increasing the morbidity as well as well as morality. early and accurate diagnosis of heart failure problem in patients of AECOPD is very difficult and challenging [26].

It was concluded from this study NT-Pro-BNP will be helpful in early diagnosis of with HF hence it will be helpful in early initiation of treatment of heart failure with medications. decreasing the hospital admissions, hospital stay and cost burden on patients and health care system. It suggests theoretical basis for sex-based clinical trials and management [27].

\section{References}

1. Patel, J. G., Nagar, S. P., \& Dalal, A. A. (2014). Indirect costs in chronic obstructive pulmonary disease: a review of the economic burden on employers and individuals in the United States. International journal of chronic obstructive pulmonary disease, 9, 289-300.

2. Hurst, J. R., Vestbo, J., Anzueto, A., Locantore, N., Müllerova, H., \& et al. (2010). Susceptibility to exacerbation in chronic obstructive pulmonary disease. New England Journal of Medicine, 363(12), 1128-1138.

3. Funk, G. C., Lang, I., Schenk, P., Valipour, A., Hartl, S., \& et al. (2008). Left ventricular diastolic dysfunction in patients with COPD in the presence and absence of elevated pulmonary arterial pressure. Chest, 133(6), 1354-1359.

4. Gale, C. P., White, J. E., Hunter, A., Owen, J., Allen, J., \& et al. (2011). Predicting mortality and hospital admission in patients with COPD: significance of NT pro-BNP, clinical and echocardiographic assessment. Journal of Cardiovascular Medicine, 12(9), 613-618.

5. Laukkanen, J. A., Kurl, S., Ala-Kopsala, M., Vuolteenaho, O., Ruskoaho, H., \& et al. (2006). Plasma N-terminal fragments of natriuretic propeptides predict the risk of cardiovascular events and mortality in middle-aged men. European heart journal, 27(10), 1230-1237.

6. Daniels, L. B., \& Maisel, A. S. (2007). Natriuretic peptides. Journal of the American college of cardiology, 50(25), 2357-2368.

7. Calzetta, L., Orlandi, A., Page, C., Rogliani, P., Rinaldi, B., \& et al. (2016). Brain natriuretic peptide: much more than a biomarker. International journal of cardiology, 221, 10311038 . 
8. Medina, A. M., Marteles, M. S., Sáiz, E. B., Martínez, S. S., Laiglesia, F. R., \& et al. (2011). Prognostic utility of NT-proBNP in acute exacerbations of chronic pulmonary diseases. European journal of internal medicine, 22(2), 167 171.

9. Cargill, R. I., \& Lipworth, B. J. (1996). Atrial natriuretic peptide and brain natriuretic peptide in cor pulmonale: hemodynamic and endocrine effects. Chest, 110(5), 1220 1225.

10. De Lemos, J. A., McGuire, D. K., \& Drazner, M. H. (2003). B-type natriuretic peptide in cardiovascular disease. The Lancet, 362(9380), 316-322.

11. Doyama, K., Fukumoto, M., Takemura, G., Tanaka, M., Oda, T., \& et al. (1998). Expression and distribution of brain natriuretic peptide in human right atria. Journal of the American college of cardiology, 32(7), 1832-1838.

12. Nishimura, K., Nishimura, T., Onishi, K., Oga, T., Hasegawa, Y., \& et al. (2014). Changes in plasma levels of B-type natriuretic peptide with acute exacerbations of chronic obstructive pulmonary disease. International journal of chronic obstructive pulmonary disease, 9, 155-162.

13. Januzzi Jr, J. L., Camargo, C. A., Anwaruddin, S., Baggish, A. L., Chen, A. A., \& et al. (2005). The N-terminal ProBNP investigation of dyspnea in the emergency department (PRIDE) study. The American journal of cardiology, 95(8), 948-954.

14. Linssen, G. C., Bakker, S. J., Voors, A. A., Gansevoort, R. T., Hillege, H. L., \& et al. (2010). N-terminal pro-B-type natriuretic peptide is an independent predictor of cardiovascular morbidity and mortality in the general population. European heart journal, 31(1), 120-127.

15. Hartmann, F., Packer, M., Coats, A. J., Fowler, M. B., Krum, H., \& et al. (2004). Prognostic impact of plasma N-terminal pro-brain natriuretic peptide in severe chronic congestive heart failure: a substudy of the Carvedilol Prospective Randomized Cumulative Survival (COPERNICUS) trial. Circulation, 110(13), 1780-1786.

16. Sabit, R., Bolton, C. E., Fraser, A. G., Edwards, J. M., Edwards, P. H., \& et al. (2010). Sub-clinical left and right ventricular dysfunction in patients with COPD. Respiratory medicine, 104(8), 1171-1178.

17. Dickstein, K., Cohen-Solal, A., Filippatos, G., McMurray, J. J., Ponikowski, P., \& et al. (2008). ESC Guidelines for the diagnosis and treatment of acute and chronic heart failure 2008: The Task Force for the Diagnosis and Treatment of Acute and Chronic Heart Failure 2008 of the European Society of Cardiology. Developed in collaboration with the Heart Failure Association of the ESC (HFA) and endorsed by the European Society of Intensive Care Medicine (ESICM). European heart journal, 29(19), 2388-2442.
18. Chang, C. L., Robinson, S. C., Mills, G. D., Sullivan, G. D., Karalus, N. C., \& et al. (2011). Biochemical markers of cardiac dysfunction predict mortality in acute exacerbations of COPD. Thorax, 66(9), 764-768.

19. Bruins, S., Fokkema, M. R., Romer, J. W., DeJongste, M. J., van der Dijs, F. P., \& et al. (2004). High intraindividual variation of B-type natriuretic peptide (BNP) and amino-terminal proBNP in patients with stable chronic heart failure. Clinical chemistry, 50(11), 2052-2058.

20. Cheng, R., Liu, C., Yang, J., Yang, Y., Chen, R., \& et al. (2021). Sex differences in the incidence and risk factors of myocardial injury in COVID-19 patients: a retrospective cohort study. Frontiers in physiology, 12, 632123.

21. El Mallawany, H., Mahmoud, M. I., Morsi, T. S., \& ELShiekh, R. M. (2014). Role of N-terminal pro B-type natriuretic peptide in acute exacerbation of chronic obstructive pulmonary disease. Egyptian Journal of Chest Diseases and Tuberculosis, 63(1), 57-65.

22. Adrish, M., Nannaka, V. B., Cano, E. J., Bajantri, B., \& Diaz-Fuentes, G. (2017). Significance of NT-pro-BNP in acute exacerbation of COPD patients without underlying left ventricular dysfunction. International journal of chronic obstructive pulmonary disease, 12, 1183-1189.

23. Patel, A. R., Kowlessar, B. S., Donaldson, G. C., Mackay, A. J., Singh, R., \& et al. (2013). Cardiovascular risk, myocardial injury, and exacerbations of chronic obstructive pulmonary disease. American journal of respiratory and critical care medicine, 188(9), 1091-1099.

24. Groenewegen, K. H., Schols, A. M., \& Wouters, E. F. (2003). Mortality and mortality-related factors after hospitalization for acute exacerbation of COPD. Chest, 124(2), 459-467.

25. Rutten, F. H., Cramer, M. J. M., Zuithoff, N. P., Lammers, J. W. J., Verweij, W., \& et al. (2007). Comparison of Btype natriuretic peptide assays for identifying heart failure in stable elderly patients with a clinical diagnosis of chronic obstructive pulmonary disease. European journal of heart failure, 9(6-7), 651-659.

26. Wheaton, A. G., Cunningham, T. J., Ford, E. S., \& Croft, J. B. (2015). Employment and activity limitations among adults with chronic obstructive pulmonary disease-United States, 2013. MMWR. Morbidity and mortality weekly report, 64(11), 289-295.

27. Roversi, S., Fabbri, L. M., Sin, D. D., Hawkins, N. M., \& Agusti, A. (2016). Chronic obstructive pulmonary disease and cardiac diseases. An urgent need for integrated care. American journal of respiratory and critical care medicine, 194(11), 1319-1336.
Copyright: (C2022 Khalida Soomro. This is an open-access article distributed under the terms of the Creative Commons Attribution License, which permits unrestricted use, distribution, and reproduction in any medium, provided the original author and source are credited. 